\title{
Desenvolvimento e reprodução da mariposa-oriental em macieira e pessegueiro
}

\author{
Oscar Arnaldo Batista Neto e Silva(1), Marcos Botton(2), Mauro Silveira Garcia(3), \\ Alexandre Zandona Bisognin ${ }^{(3)}$ e Dori Edson Nava(4)
}

\begin{abstract}
(1)Escola Superior de Agricultura Luiz de Queiroz, Departamento de Entomologia e Acarologia, Avenida Pádua Dias, no 11, CEP 13418-900 Piracicaba, SP. E-mail: oscar.netto@usp.br (2)Embrapa Uva e Vinho, Caixa Postal 130, CEP 95700-000 Bento Gonçalves, RS. E-mail: marcos@cnpuv.embrapa.br (3)Universidade Federal de Pelotas, Faculdade de Agronomia Eliseu Maciel, Departamento de Fitossanidade, Caixa Postal 354, CEP 96010-900 Pelotas, RS. E-mail: garciasmauro@yahoo.com.br, alexandrezbisognin@yahoo.com.br ${ }^{(4)}$ Embrapa Clima Temperado, Caixa Postal 403, CEP 96001-970 Pelotas, RS. E-mail: nava.dori@cpact.embrapa.br
\end{abstract}

Resumo - O objetivo deste trabalho foi avaliar o desenvolvimento de estágios imaturos e parâmetros reprodutivos de adultos de Grapholita molesta em dietas naturais, constituídas de ramos e frutos de pessegueiro 'Vanguarda', frutos de macieira 'Gala' e 'Fuji', e em dieta artificial à base de milho. O delineamento experimental foi inteiramente casualizado. Considerou-se cada inseto uma repetição e, no estudo dos parâmetros biológicos dos adultos, cada casal foi considerado uma repetição. Foi calculada a tabela de vida de fertilidade. $\mathrm{O}$ tempo para o desenvolvimento das lagartas foi menor em ramos e frutos do pessegueiro, em comparação a frutos de macieira e dieta à base de farinha de milho. Quanto à sobrevivência das larvas, não houve diferença entre as dietas. Com base na tabela de vida de fertilidade, os frutos da cultivar Fuji foram mais adequados ao desenvolvimento de G. molesta do que os da Gala. Os ramos de pessegueiro foram mais adequados ao desenvolvimento do inseto do que os frutos. A dieta à base de farinha de milho foi adequada para a criação dos insetos em laboratório. As dietas avaliadas afetam a taxa de sobrevivência, o desenvolvimento e o potencial de crescimento populacional de G. molesta.

Termos para indexação: Grapholita molesta, Malus domestica, Prunus persica, criação em laboratório, tabela de vida.

\section{Development and reproduction of the oriental fruit moth in apple and peaches}

\begin{abstract}
The objective of this work was to evaluate the immature stages development and the reproductive parameters of Grapholita molesta adults reared on shoots and fruit of 'Vanguarda' peach, on fruit of 'Gala' and 'Fuji' apples, and on a diet formulated with corn meal. The experimental design was completely randomized. Each insect was considered one replicate; and for the biological parameters of adults study, each couple was considered a replicate. The fertility life table was calculated. The time for larvae development was shorter in peach shoots and fruits, in comparison with apple fruit and corn meal diets. There were no differences for larvae survival, in the comparison between diets. Based on fertility life table, 'Fuji' apple fruit are more appropriated to the G. molesta development than Gala ones. Peach shoots were more suited for insect development than fruit. The corn meal-based diet is suitable to rear G. molesta in laboratory. The evaluated diets affect survival rate, development and growth potential of $G$. molesta population.
\end{abstract}

Index terms: Grapholita molesta, Malus domestica, Prunus persica, laboratory rearing, life table.

\section{Introdução}

A mariposa-oriental Grapholita molesta (Busck, 1916) (Lepidoptera: Tortricidae) é uma das principais pragas da macieira Malus domestica Borkhausen e do pessegueiro Prunus persica (L.) Batsch no Brasil (Reis Filho et al., 1988; Salles, 2001; Botton et al., 2003; Monteiro \& Hickel, 2004). Os danos provocados pelo inseto são ocasionados pela alimentação das lagartas que, ao eclodir, perfuram os ramos novos e os frutos, construindo galerias (Salles, 2001). Em pessegueiro, os maiores danos são observados em pomares com plantas novas e em cultivares de ciclo tardio (Botton et al., 2003). Na macieira, os principais danos são observados em cultivares tardias, principalmente a Fuji (Myers et al., 2006c).

Embora G. molesta esteja presente no Brasil há meio século e seja uma praga prejudicial para as culturas da macieira e do pessegueiro, há lacunas em relação a informações sobre a sua biologia. Quanto ao pessegueiro, há necessidade de dados sobre o efeito do uso para alimentação dos insetos

Pesq. agropec. bras., Brasília, v.45, n.10, p.1082-1088, out. 2010 
das diferentes estruturas vegetais sobre a biologia da espécie. Na cultura da macieira, o conhecimento do comportamento reprodutivo de G. molesta, quanto aos frutos das duas principais cultivares produzidas no Brasil (Gala e Fuji) pode auxiliar na compreensão do potencial de dano à cultura.

Recentemente, uma nova metodologia foi desenvolvida para a criação do inseto em laboratório com uso de dieta artificial à base de farinha de milho (Arioli, 2007). No entanto, faltam informações sobre o desempenho reprodutivo da espécie quando criada com dieta artificial à base de milho, em comparação ao seu desempenho quando criada com alimentação natural. Assim, é importante o conhecimento do efeito do alimento no desenvolvimento e capacidade reprodutiva de G. molesta, pois isto poderá fornecer subsídios para formular estratégias de controle desta praga nas culturas da macieira e do pessegueiro.

O objetivo deste trabalho foi avaliar o desenvolvimento de estágios imaturos e parâmetros reprodutivos de adultos de G. molesta em dietas naturais, constituídas de ramos e frutos de pessegueiro 'Vanguarda', frutos de macieira 'Gala' e 'Fuji', e em dieta artificial à base de milho.

\section{Material e Métodos}

Os experimentos foram realizados no Laboratório de Biologia de Insetos e Controle Biológico, da Faculdade de Agronomia Eliseu Maciel, da Universidade Federal de Pelotas (UFPel), à $25 \pm 1^{\circ} \mathrm{C}$, com umidade relativa do ar de $70 \pm 10 \%$ e fotófase de 16 horas. Os insetos utilizados nos experimentos foram obtidos da criação de manutenção do laboratório, realizada conforme Arioli (2007), com modificação: a levedura de cerveja foi substituída por fermento biológico, na dieta artificial utilizada para o desenvolvimento larval. A criação de laboratório foi iniciada com insetos coletados em frutos de maçã, em fevereiro em 2005, de pomares comercias localizados no Município de Vacaria, RS.

O desenvolvimento de G. molesta foi estudado em dietas naturais, constituídas de ramos e frutos de pessegueiro da cultivar Vanguarda e de frutos de macieira das cultivares Gala e Fuji, e comparado com o desenvolvimento em dieta artificial à base de farinha de milho, utilizada para a criação em laboratório.

Os frutos e os ramos de pessegueiro foram coletados e utilizados no mesmo dia, durante o mês de outubro, em plantas da cultivar Vanguarda com seis anos de idade do pomar do Centro Agropecuário da Palma, da UFPel, em Capão do Leão, RS. As maçãs das cultivares Gala e Fuji foram obtidas da Cooperativa Agropecuária Pradense, no Município de Antônio Prado, RS, colhidas em março e abril e mantidas em câmara frigorífica por seis a sete meses. Foram utilizadas maçãs de tamanho uniforme, com diâmetro médio de 4 a $6 \mathrm{~cm}$, e ramos de pessegueiro de até $12 \mathrm{~cm}$. Os ramos de pessegueiro foram colocados individualmente em copos de $180 \mathrm{~mL}$, com 7,5 cm de altura x 6,5 cm de diâmetro, que continham ágar/água à $1 \%$, para sustentar o ramo em posição vertical, tendo-se utilizado como tampa outro copo em posição invertida, com capacidade de $200 \mathrm{~mL}$, com $6 \mathrm{~cm}$ de altura x $7 \mathrm{~cm}$ de diâmetro. Os pêssegos e as maçãs foram colocados individualmente em copos de plástico transparente, com capacidade de $300 \mathrm{~mL}$, com $11 \mathrm{~cm}$ de altura $\mathrm{x}$ $7,5 \mathrm{~cm}$ de diâmetro. $\mathrm{O}$ alimento foi trocado, em caso de apodrecimento dos frutos ou da necessidade de ramos adicionais para o completo desenvolvimento larval.

Em dieta artificial, as lagartas foram colocadas individualmente em tubos de vidro de fundo chato ( $8,5 \mathrm{~cm}$ de comprimento x $2,5 \mathrm{~cm}$ de diâmetro), que continham $12 \mathrm{~g}$ de dieta artificial, tampados com algodão hidrófobo.

Lagartas de até 24 horas de idade foram transferidas para as dietas natural e artificial, com auxílio de um pincel de ponta fina. Dez dias após a inoculação, foi colocado um chumaço de algodão hidrófilo junto aos órgãos vegetais, que serviu de substrato para a pupa. Foram utilizadas 160 lagartas, no tratamento dieta artificial, 150 para cada variedade de maçã e 200 para frutos e ramos de pessegueiro, e cada lagarta representou uma repetição.

Para cada tratamento, foram avaliados os seguintes parâmetros biológicos: duração e sobrevivência dos estágios de desenvolvimento, peso de pupas com 24 horas, razão sexual, duração dos períodos de pré-oviposição e oviposição, longevidade de adultos e fecundidade (diária e total). A razão sexual foi calculada pela fórmula: $r s=n^{0}$ de fêmeas $/\left(n^{\circ}\right.$ de fêmeas $+n^{\circ}$ de machos).

A longevidade e a fecundidade foram avaliadas em 20 casais com menos de 24 horas de idade, colocados individualmente em gaiolas de $\mathrm{PVC}$, com $15 \mathrm{~cm}$ de altura x $15 \mathrm{~cm}$ de diâmetro, revestidas internamente com plástico liso (substrato de oviposição) e fechadas

Pesq. agropec. bras., Brasília, v.45, n.10, p.1082-1088, out. 2010 
na parte superior com tecido tipo tule. Os adultos foram alimentados com uma solução de mel a $15 \%$, fornecida por capilaridade em tubos de $30 \mathrm{~mL}$. Diariamente, a mortalidade dos adultos e a contagem do número de ovos foram registradas, para a determinação da fecundidade. Para a determinação da fertilidade (número de ovos férteis) e do período embrionário, foram obtidos, de cada casal, dez ovos do terceiro dia de postura, que totalizaram 200 ovos por tratamento. Os ovos foram acondicionados em tubos de vidro de fundo chato, com $8,5 \mathrm{~cm}$ de altura x 2,5 cm de diâmetro. No interior dos tubos, foi colocado um pedaço de papel filtro $(2 \times 1 \mathrm{~cm})$, umedecido diariamente com água destilada; e os tubos foram fechados na parte superior com filme plástico. As posturas foram observadas diariamente.

Utilizou-se o delineamento experimental inteiramente casualizado, e cada inseto foi considerado uma repetição. Para a avaliação dos parâmetros biológicos do adulto, cada casal foi considerado uma repetição. Após a análise de variância, as médias foram comparadas pelo teste de Tukey a $5 \%$ de probabilidade, com o uso do programa SAS (SAS Institute, 2000). Os resultados relativos à razão sexual foram analisados pelo teste de qui-quadrado $\left(\chi^{2}\right)$, também a $5 \%$ de probabilidade.

Foi calculada a tabela de vida de fertilidade dos insetos nas diferentes dietas, tendo-se estimado a duração média de uma geração $(\mathrm{T})$, a taxa líquida de reprodução (Ro) e as razões de aumento infinitesimal $(\mathrm{Rm})$ e finita $(\lambda)$. Os parâmetros da tabela de vida de fertilidade e seus respectivos erros-padrão foram estimados e as médias foram comparadas pelo teste $\mathrm{t}$ bilateral, a $5 \%$ de probabilidade, por meio da programação lifetable.sas (Maia et al., 2000) do programa estatístico SAS (SAS Institute, 2000).

\section{Resultados e Discussão}

As dietas naturais (frutos e ramos) e a artificial, à base de milho, interferiram significativamente nos parâmetros biológicos de G. molesta (Tabela 1). O tempo de desenvolvimento das lagartas foi menor em ramos e frutos do pessegueiro, em comparação aos frutos de macieira e dieta artificial. Estes resultados estão de acordo com Myers et al. (2007), que afirmam que as lagartas de G. molesta completam seu desenvolvimento de forma mais rápida tanto em frutos quanto em ramos de pessegueiro do que em maçãs. Em pessegueiro, a duração do estágio larval foi mais rápida em ramos do que em frutos. A maioria das lagartas necessitou de três a quatro ramos para completar seu desenvolvimento, enquanto as que foram criadas em frutos completaram o estágio em somente uma estrutura vegetal. Isto confirma observações de Myers et al. (2006b), realizadas em campo, de que uma lagarta de $G$. molesta pode danificar mais de um ramo de pessegueiro durante o seu desenvolvimento. Nos frutos de macieira, as lagartas levaram menos tempo para completar este estágio na cultivar Gala do

Tabela 1. Média \pm erro-padrão dos parâmetros biológicos de Grapholita molesta mantida em dietas naturais (ramos e frutos de pessegueiro 'Vanguarda' e frutos de maçã 'Gala' e 'Fuji') e em dieta artificial à base de farinha de milho, em laboratório(1).

\begin{tabular}{|c|c|c|c|c|c|c|c|c|}
\hline Parâmetros biológicos ${ }^{(2)}$ & Dieta artificial & Ramos de pessegueiro & Pêssego 'Vanguarda' & Maçã 'Fuji' & Maçã 'Gala' & G.L. & Valor F & $\mathrm{P}$ \\
\hline Estágio larval (dias) & $15,5 \pm 0,11 \mathrm{c}$ & $13,0 \pm 0,17 \mathrm{e}$ & $14,0 \pm 0,17 \mathrm{~d}$ & $17,3 \pm 0,24 \mathrm{a}$ & $16,3 \pm 0,22 b$ & 4 & 91,93 & $<0,0001$ \\
\hline Sobrevivência larval (\%) & $79,6 \pm 9,10 \mathrm{a}$ & $55,3 \pm 11,65 b c$ & $42,5 \pm 11,84 \mathrm{c}$ & $57,2 \pm 7,82 \mathrm{~b}$ & $46,5 \pm 12,24 \mathrm{bc}$ & 4 & 17,06 & $<0,0001$ \\
\hline Estágio de pupa (dias) & $6,4 \pm 0,12 \mathrm{bc}$ & $7,0 \pm 0,15 \mathrm{ab}$ & $7,2 \pm 0,18 \mathrm{a}$ & $6,0 \pm 0,21 \mathrm{c}$ & $6,1 \pm 0,20 \mathrm{c}$ & 4 & 9,06 & $<0,0001$ \\
\hline Sobrevivência pupal (\%) & $95,5 \pm 3,75 \mathrm{a}$ & $76,7 \pm 14,42 b$ & $92,8 \pm 5,83 \mathrm{a}$ & $96,6 \pm 7,15 \mathrm{a}$ & $86,6 \pm 11,51 \mathrm{ab}$ & 4 & 6,79 & $<0,0002$ \\
\hline Lagarta a adulto (dias) & $22,1 \pm 0,14 b$ & $20,0 \pm 0,19 \mathrm{~d}$ & $21,2 \pm 0,20 \mathrm{c}$ & $23,7 \pm 0,32 \mathrm{a}$ & $22,5 \pm 0,30 \mathrm{~b}$ & 4 & 34,93 & $<0,0001$ \\
\hline Sobrevivência Lagarta-adulto (\%) & $75,0 \pm 11,56 \mathrm{a}$ & $38,2 \pm 8,54 \mathrm{bc}$ & $36,5 \pm 9,94 \mathrm{c}$ & $52,3 \pm 10,50 \mathrm{~b}$ & $37,5 \pm 11,21 \mathrm{c}$ & 4 & 20,87 & $<0,0001$ \\
\hline Razão sexual & $0,43^{\text {ns }}$ & $0,51^{\mathrm{ns}}$ & $0,44^{\mathrm{ns}}$ & $0,39^{\text {ns }}$ & $0,45^{\mathrm{ns}}$ & 4 & - & - \\
\hline Peso de pupas (mg) & $13,9 \pm 0,29 \mathrm{a}$ & $9,6 \pm 0,32 \mathrm{c}$ & $11,2 \pm 0,33 b$ & $12,3 \pm 0,49 b$ & $11,1 \pm 0,33 \mathrm{~b}$ & 4 & 25,55 & $<0,0001$ \\
\hline Pré-oviposição (dias) & $2,9 \pm 0,25 \mathrm{bc}$ & $3,7 \pm 0,22 \mathrm{ab}$ & $4,8 \pm 0,47 \mathrm{a}$ & $2,2 \pm 0,18 \mathrm{c}$ & $2,6 \pm 0,24 \mathrm{bc}$ & 4 & 12,78 & $<0,0001$ \\
\hline Oviposição (dias) & $14,4 \pm 0,66 \mathrm{a}$ & $12,4 \pm 0,92 \mathrm{ab}$ & $10,5 \pm 1,00 \mathrm{bc}$ & $11,8 \pm 0,53 \mathrm{ab}$ & $8,3 \pm 0,59 \mathrm{c}$ & 4 & 8,58 & $<0,0001$ \\
\hline Longevidade dos adultos (dias) & $22,9 \pm 0,41 \mathrm{a}$ & $20,6 \pm 0,82 \mathrm{ab}$ & $19,4 \pm 0,95 b$ & $18,7 \pm 0,62 b$ & $15,2 \pm 0,52 \mathrm{c}$ & 4 & 15,65 & $<0,0001$ \\
\hline Fecundidade diária (ovos) & $8,4 \pm 1,99$ & $10,3 \pm 2,29$ & $9,2 \pm 1,67$ & $13,3 \pm 3,11$ & $13,5 \pm 2,85$ & 4 & 0,93 & $<0,4486$ \\
\hline Fecundidade total (ovos) & $139,7 \pm 7,37 b c$ & $144,6 \pm 9,65 b c$ & $110,1 \pm 9,36 \mathrm{c}$ & $202,2 \pm 12,49 \mathrm{a}$ & $152,8 \pm 7,82 b$ & 4 & 12,31 & $<0,0001$ \\
\hline Período embrionário (dias) & $3,2 \pm 0,15 \mathrm{a}$ & $3,6 \pm 0,13 \mathrm{a}$ & $3,8 \pm 0,10 \mathrm{a}$ & $3,5 \pm 0,19 \mathrm{a}$ & $3,5 \pm 0,12 \mathrm{a}$ & 4 & 1,99 & $<0,1057$ \\
\hline Viabilidade de ovos (\%) & $86,7 \pm 10,25 \mathrm{a}$ & $83,9 \pm 14,21 \mathrm{a}$ & $76,5 \pm 11,14 \mathrm{a}$ & $80,0 \pm 14,54 a$ & $77,3 \pm 15,27 \mathrm{a}$ & 4 & 1,43 & $<0,2343$ \\
\hline
\end{tabular}

${ }^{(1)}$ Médias seguidas de letras iguais, nas linhas, não diferem entre si pelo teste de Tukey, a $5 \%$ de probabilidade. ${ }^{(2)}$ Temperatura de $25 \pm 1^{\circ} \mathrm{C}$; umidade relativa de $70 \pm 10 \%$; fotófase de 16 horas. ${ }^{\text {ns }}$ Não significativo pelo teste do qui-quadrado $\left(\chi^{2}\right)$ a $5 \%$ de probabilidade. $\mathrm{P}$, significância estatística do teste $\mathrm{F}$. 
que na Fuji (1 dia, em média). Os valores registrados foram próximos aos 16,6 dias observados por Rosenthal et al. (1994), em maçã 'Fuji'.

No caso da dieta artificial, os insetos levaram, em média, 15,5 dias para atingir o estágio de pupa, inferior aos 19,2 dias encontrados por Arioli (2007). No entanto, na dieta de Arioli (2007), foi utilizada levedura no lugar de fermento biológico, o que pode ser um fator determinante na duração do estágio de lagarta.

Aduração do estágio pupal diferiu significativamente, tendo sido maior para insetos criados nas estruturas vegetais do pessegueiro, em comparação aos criados em maçãs. Em comparação aos insetos que se alimentaram de dieta artificial, somente os que se alimentaram de frutos de pessegueiro tiveram maior duração do estágio de pupa.

Quando considerada de forma conjunta, a duração do período lagarta-adulto foi menor nos insetos criados em ramos e frutos de pessegueiro e diferiu significativamente da duração dos criados nas maçãs (Tabela 1), provavelmente em razão da menor duração da fase larval. Os insetos que se alimentaram com frutos da cultivar Gala atingiram o estágio adulto em menos tempo do que os que se alimentaram com frutos da Fuji. Os valores registrados neste trabalho foram inferiores aos 27 e 25,3 dias obtidos por Rosenthal et al. (1994), que criaram G. molesta em maçã 'Fuji' e em marmelo, Cydonia oblonga Miller (Rosaseae), respectivamente, à temperatura de $26^{\circ} \mathrm{C}$. Quanto a esse parâmetro, a dieta artificial proporcionou valor intermediário (22,1 dias) aos alimentos naturais, e inferior ao encontrado por Arioli (2007).

O maior peso de pupas foi obtido quando os insetos foram criados em dieta artificial $(13,9 \mathrm{mg})$ e o menor foi obtido nos insetos que se desenvolveram sobre ramos de pessegueiro $(9,6 \mathrm{mg})$. Esta diferença pode estar relacionada à qualidade do alimento, que depende de atributos físicos que influenciam a capacidade do inseto de consumir e digerir o alimento, além de aleloquímicos e componentes nutricionais. O peso de pupas na comparação entre as dietas de maçãs não apresentou diferença significativa, e os valores foram próximos ao encontrado por Rosenthal et al. (1994) em maçã 'Fuji' (11 mg) e Marín et al. (2006) em maçãs verdes (10 mg).

Quanto à razão sexual, os valores obtidos variaram entre 0,51 (ramos de pessegueiro) a 0,39 (maçã 'Fuji'). Contudo, esta diferença não foi significativa, o que indica que os alimentos fornecidos não alteraram a proporção de machos e fêmeas na espécie, e os valores estiveram próximos a 0,5 , mencionado por Salles (2001).

A maior sobrevivência larval (75\%) foi registrada em insetos criados com dieta artificial (Tabela 1). Esses valores foram inferiores aos 95,3\% encontrados por Arioli (2007), porém próximos aos 76 e 71,4\% registrados por Grellmann et al. (1991) e Rosenthal et al. (1994), respectivamente, que utilizaram a dieta artificial sugerida por Ivaldi-sender (1974). Não houve diferença significativa entre as dietas de frutos e ramos de pessegueiro, bem como não foi observada diferença entre as dietas de cultivares de maçã Gala e Fuji. Em trabalhos conduzidos no campo, Myers et al. (2006b) demonstraram diferenças na sobrevivência larval, quando compararam diferentes cultivares de maçã, em que a Fuji permitiu a maior sobrevivência larval. Rosenthal et al. (1994) encontraram valores superiores para larvas, quando criadas com maçã 'Fuji' $(71,2 \%)$ ou marmelo $(78,7 \%)$. De acordo com Myers et al. (2006b), em frutos de macieira, a sobrevivência de G. molesta é dependente essencialmente da capacidade de a lagarta penetrar no fruto, pois a mortalidade no interior do fruto é baixa. O tempo gasto pela lagarta para penetrar no fruto também pode variar entre as cultivares, e é de 6 a 14 horas para a Fuji (Myers et al., 2006c).

A sobrevivência do estágio de pupa foi afetada negativamente, quando os insetos se desenvolveram sobre ramos de pessegueiro. Os demais alimentos não diferiram entre si. A maior mortalidade de pupas de insetos que se alimentaram de ramos de pessegueiro pode ter relação com o baixo ganho de peso dessas pupas, as quais poderiam não ter alcançado o peso ideal e poderiam ter poucas reservas energéticas para os eventos de metamorfose (histólise e histogênese).

A sobrevivência no período lagarta-adulto foi menor nos insetos criados em frutos e ramos de pessegueiro, em comparação aos criados em dieta artificial. Esta diferença tem forte influência da sobrevivência larval, já que o valor obtido para os insetos criados em dieta artificial, para esse parâmetro, é quase o dobro do observado para os insetos que se alimentaram de frutos de pêssego. Para que uma dieta seja considerada satisfatória para a criação de insetos em laboratório, Singh (1983) preconiza que seja alcançado pelo menos $75 \%$ de sobrevivência no período de lagarta-adulto. 
No presente estudo, a dieta avaliada atendeu a esta exigência. Quando G. molesta foi criada em pêssego e em ramos de pessegueiro, não foi observada diferença significativa na sobrevivência no período lagarta-adulto. Entre as cultivares de maçã, a Fuji proporcionou maior sobrevivência no período lagarta-adulto do que a Gala.

Os parâmetros reprodutivos de G. molesta foram alterados em consequência da alimentação das lagartas (Tabela 1). O período de pré-oviposição foi maior nos insetos criados em frutos e ramos de pessegueiro, em comparação aos frutos da maçã 'Fuji'. A duração deste período, nos insetos que se alimentaram de dieta artificial, diferiu somente dos que se alimentaram de frutos de pessegueiro. $O$ atraso para a cópula também é um fator que pode interferir no período de pré-oviposição de G. molesta. De acordo com Fraser $\&$ Trimble (2001), o período de pré-oviposição é maior nas fêmeas copuladas com 24 horas após a emergência do que em fêmeas copuladas de dois a cinco dias após a emergência. Os insetos que se desenvolveram sobre maçã 'Gala' apresentaram o menorperíodo de oviposição observado entre as dietas avaliadas. Os insetos criados em dieta artificial, em ramos de pessegueiro e em maçã 'Fuji' não diferiram entre si.

Adultos de insetos que se alimentaram de dieta artificial apresentaram longevidade equivalente à de insetos criados sobre ramos de pessegueiro. Quando se compararam as cultivares de maçã, foi observado que os adultos de insetos que se alimentaram da 'Gala' foram menos longevos do que os insetos criados em frutos da 'Fuji'. De acordo com Myers et al. (2006b), futuramente, os limiares de ação, baseados em captura de adultos de G. molesta com armadilhas de feromônio sexual, poderão variar em razão da cultivar, quando se considerarem as taxas de sobrevivência e desenvolvimento diferenciado da espécie entre as cultivares. Quando se avaliou a flutuação populacional dos adultos de G. molesta, em pomares de maçã da cultivar Fuji, observou-se que os vôos nesta cultivar podem ter maior duração, pois os adultos foram mais longevos do que os insetos que se alimentaram de maçã 'Gala'. Contudo, essa hipótese necessita ser validada em experimentos com este objetivo.

A fecundidade diária não variou em consequência do alimento utilizado, o que indica que G. molesta manteve o mesmo ritmo de postura, independentemente do alimento fornecido às lagartas (Tabela 1).
Em contrapartida, o número médio de ovos por fêmea variou de 110 em frutos de pessegueiro a $202 \mathrm{em}$ maçã 'Fuji'. Myers et al. (2006a) também registraram que a fecundidade de G. molesta, mantida sobre frutos de maçãs, é o dobro da observada em frutos de pessegueiro.

No caso do pessegueiro, os insetos que se alimentaram dos frutos não apresentaram uma fecundidade diferente daqueles criados em ramos (Tabela 1). Em frutos de maçã, os insetos que se desenvolveram sobre a cultivar Gala ovipositaram em média 50 ovos a menos por fêmea, em comparação ao obtido na cultivar Fuji. Diferenças na oviposição também foram observadas em insetos que se desenvolveram sobre maçã, por Tzanakakis \& Phillips (1969), Alfonso \& Marín (2004) e Marín et al. (2006), que observaram variação de 126 a 160 ovos por fêmeas. Arioli (2007) registrou 234 ovos por fêmea na dieta à base de milho, enquanto no presente trabalho foram obtidos 139 ovos.

A duração do período embrionário foi, em média, de 3,5 dias, com viabilidade de ovos de $80,9 \%$, sem diferença significativa em consequência do alimento fornecido (Tabela 1).

Com relação aos parâmetros de crescimento populacional, com base na tabela de vida de fertilidade, foram observadas diferenças na adequação entre a dieta artificial, as cultivares de maçã e as estruturas vegetais do pessegueiro (Tabela 2).

Uma análise conjunta dos dados permite constatar que a cultivar Fuji apresentou maiores valores de Ro, $\mathrm{r}_{\mathrm{m}}$, e $\lambda$, em comparação à maçã 'Gala' e ao fruto de pessegueiro, o que indica que esta cultivar propicia melhores condições de aumento populacional de G. molesta. Este fato indica que, em regiões onde os pomares de pessegueiro se encontram próximos aos

Tabela 2. Duração das gerações (T), taxa líquida de reprodução (Ro), razão infinitesimal de aumento (rm) e razão finita de aumento $(\lambda)$ para Grapholita molesta mantida em diferentes alimentos, em laboratório, sob condições controladas de temperatura $\left(25 \pm 1^{\circ} \mathrm{C}\right)$, umidade relativa do ar $(70 \pm 10 \%)$ e fotófase (16 horas) $)^{(1)}$.

\begin{tabular}{lcrcc}
\hline Alimento & T (dias) & \multicolumn{1}{c}{ Ro } & $\mathrm{rm}$ & \multicolumn{1}{c}{$\lambda$} \\
\hline Dieta artificial & $26,2 \pm 0,22 \mathrm{a}$ & $37,8 \pm 2,00 \mathrm{a}$ & $0,1388 \pm 0,002 \mathrm{~b} 1,1487 \pm 0,003 \mathrm{~b}$ \\
Ramos de pessegueiro & $23,8 \pm 0,16 \mathrm{~b}$ & $23,57 \pm 1,57 \mathrm{~b}$ & $0,1326 \pm 0,003 \mathrm{~b}$ & $1,1418 \pm 0,003 \mathrm{~b}$ \\
Pêssego 'Vanguarda' & $25,9 \pm 0,25 \mathrm{a}$ & $14,16 \pm 1,22 \mathrm{c}$ & $0,1019 \pm 0,003 \mathrm{c} 1,1074 \pm 0,004 \mathrm{c}$ \\
Maçã 'Fuji' & $23,7 \pm 0,17 \mathrm{~b}$ & $32,68 \pm 2,02 \mathrm{a}$ & $0,1473 \pm 0,003 \mathrm{a} 1,1587 \pm 0,004 \mathrm{a}$ \\
Maçã 'Gala' & $21,9 \pm 0,18 \mathrm{c}$ & $20,30 \pm 1,10 \mathrm{~b}$ & $0,1376 \pm 0,002 \mathrm{~b} 1,1475 \pm 0,003 \mathrm{~b}$ \\
\hline \multicolumn{3}{c}{ (1) Médias seguidas de letras iguais nas colunas, não diferem entre si pelo } \\
teste t bilateral, a 5\% de probabilidade.
\end{tabular}


de macieira, os pomares de pessegueiro podem ser mais atacados, em razão da migração da população das áreas de macieira na primavera (pós diapausa), o que pode exigir um manejo diferenciado para a praga nessa situação, conforme já salientado por Myers et al. (2006c).

Quando as cultivares de maçã foram comparadas, verificou-se que a Fuji, além de ser mais tardia, o que em hipótese permite um maior número de gerações da praga, mostrou-se mais adequada ao desenvolvimento do inseto do que a Gala. Na comparação entre as estruturas vegetais do pessegueiro, os ramos mostraramse mais adequados ao desenvolvimento do inseto, uma vez que os parâmetros Ro, $\mathrm{r}_{\mathrm{m}}$ e $\lambda$ foram maiores quando os insetos foram criados sobre este alimento. Com esse efeito diferencial no desenvolvimento do inseto, quanto maior o período com disponibilidade de ramos para a multiplicação da espécie, em hipótese, maiores serão as chances de números elevados da praga na cultura. Em outra situação, o incremento populacional, como consequência da alimentação sobre ramos, poderá afetar o manejo do inseto nos lugares em que a macieira e o pessegueiro são cultivados próximos. Nesse caso, na ausência de barreira física, pode haver a migração de adultos dos pomares de pessegueiro para cultivares de macieira de ciclo tardio (Poltronieri et al., 2008).

Os insetos que se alimentaram de dieta artificial tiveram o parâmetro Ro superior e os parâmetros $r_{m}$ e $\lambda$ equivalentes aos obtidos com maçã 'Gala'. Isso indica que a dieta artificial à base de farinha de milho permitiu obter insetos biologicamente comparáveis aos criados com alimento natural. Assim, a dieta artificial à base de farinha de milho, modificada de Arioli (2007), confirmou seu potencial para a criação de $G$. molesta em laboratório.

\section{Conclusões}

1. O tempo para o desenvolvimento das lagartas de Grapholita molesta é menor em ramos e frutos do pessegueiro do que em frutos de macieira.

2. Não há diferença, quanto à sobrevivência larval, entre frutos e ramos de pessegueiro e de macieira.

3. Grapholita molesta apresenta maior crescimento populacional quando se desenvolve em frutos da cultivar de maçã Fuji do que nas demais estruturas vegetais de pessegueiro e macieira.
4. Maçãs da cultivar Fuji são mais adequadas ao desenvolvimento de $G$. molesta do que as da cultivar Gala.

5. Os ramos de pessegueiro são mais adequados ao desenvolvimento de $G$. molesta do que os frutos.

6. A dieta artificial à base de farinha de milho é adequada para a criação de G. molesta em laboratório.

\section{Agradecimentos}

Ao Conselho Nacional de Desenvolvimento Cientifico e Tecnológico, pela concessão de bolsas; à Cooperativa Agropecuária Pradense, pela doação das maçãs utilizadas nos experimentos; e ao professor Dr. José Carlos Fachinello, pela doação dos ramos e frutos de pessegueiro.

\section{Referências}

ALFONSO, A.M.; MARÍN, M.S. Grapholita molesta en condiciones de laboratorio: evaluación de la relación de sexos. Revista de la Facultad de Ciencias Agrarias, v.36, p.23-29, 2004.

ARIOLI, A. Técnica de criação e controle de Grapholita molesta (Busck, 1916) (Lepidoptera: Tortricidae) na cultura da macieira. 2007. 83p. Tese (Doutorado) - Universidade Federal de Pelotas, Pelotas.

BOTTON, M.; ARIOLI, C.J.; BAVARESCO, A.; SCOZ, P.L. Principais pragas do pessegueiro. In: GARRIDO, L. da C.; BOTTON, M. (Org.). Sistema de produção de pêssego de mesa na Região da Serra Gaúcha. Bento Gonçalves: Embrapa Uva e Vinho, 2003. p.1-16. (Embrapa Uva e Vinho. Sistemas de produção, 3).

FRASER, H.W.; TRIMBLE, R.M. Effect of delayed mating on reproductive biology of the Oriental fruit moth (Lepidoptera: Tortricidae). Canadian Entomologist, v.133, p.219-227, 2001.

GRELLMANN E.O; LOECK, A.E.; SALLES L.A.B.; FACHINELLO, J.C. Ciclo evolutivo de Grapholita molesta (Busck, 1916) (Lepidoptera: Olethreutidae) em diferentes temperaturas. Revista Brasileira de Fruticultura, v.13, p.21-26, 1991.

IVALDI-SENDER, C. Techniques simples pour un élevage permanent de la tordeuse orientale, Grapholita molesta (Lepidoptera: Tortricidae) sur milieu artificiel. Annales de Zoologie et Ecologie Animales, v.6, p.337-343, 1974.

MAIA, A. de H.N.; LUIZ, A.J.B.; CAMPANHOLA, C. Statistical inference on associated fertility life table parameters using jackknife technique: computational aspects. Journal of Economic Entomology, v.93, p.511-518, 2000.

MARÍN, M.S.; SÁEZ, C.C.; CABALLERO, E.A.; QUERCETTI, M.J. Grapholita molesta: caracterización de una cría artificial. Revista de la Facultad de Ciencias Agrarias, v.38, p.7-12, 2006. 
MONTEIRO, L.B.; HICKEL, E. Pragas de importância econômica em fruteiras de caroço. In: MONTEIRO, L.B.; DE MIO, L.L.M.; SERRAT, B.M.; MOTA,A.C.V.; CUQUEL, F.L.(Org.) Fruteiras de caroço: uma visão ecológica. Curitiba: UFPR, 2004. p.223-264.

MYERS, C.T.; HULL, L.A.; KRAWCZYK, G. Comparative survival rates of Oriental fruit moth (Lepidoptera: Tortricidae) larvae on shoots and fruit of apple and peach. Journal of Economic Entomology, v.99, p.1299-1309, 2006b.

MYERS, C.T.; HULL, L.A.; KRAWCZYK, G. Early-season host plant fruit impacts on reproductive parameters of the Oriental fruit moth (Lepidoptera: Tortricidae). Journal of Entomological Science, v.41, p.65-74, 2006a.

MYERS, C.T.; HULL, L.A.; KRAWCZYK, G. Effects of orchard host plants (apple and peach) on development of Oriental fruit moth (Lepidoptera: Tortricidae). Journal of Economic Entomology, v.100, p.421-430, 2007.

MYERS, C.T.; HULL, L.A.; KRAWCZYK, G. Seasonal and cultivar associated variation in the oviposition preference of Oriental fruit moth (Lepidoptera: Tortricidae) adults and feeding behavior of neonate larvae in apples. Journal of Economic Entomology, v.99, p.349-358, 2006 c.
POLTRONIERI, A.S.; MONTEIRO, L.B.; SCHUBER, J.M. Prospecção da diapausa da mariposa-oriental no período de dormência do pessegueiro. Scientia Agraria, v.9, p.67-72, 2008.

REIS FILHO, W.; NORA, I.; MELZER, R. Population dynamics of Grapholita molesta (Busck, 1916) and its adaptation on apple in South Brazil. Acta Horticulturae, n.232, p.202-208, 1988.

ROSENTHAL, M. de.A.; LOECK,A.E.; SILVAJUNIOR, P. Criação de Grapholita molesta (Busck, 1916) (Lepidoptera: Olethreutidae) em dietas artificiais e naturais. Pesquisa Agropecuária Brasileira, v.29, p.1847-1853, 1994.

SALLES, L.A.B. de. A mariposa-oriental, Grapholita molesta (Lepidoptera: Tortricidae). In: VILELA, E.F.; ZUCCHI, R.A.; CANTOR, F. (Ed.). Histórico e impacto das pragas introduzidas no Brasil. Ribeirão Preto: Holos, 2001. p.42-45.

SAS INSTITUTE. Statistical analysis system: getting started with the SAS learning. Cary: SAS Institute, 2000. 81p.

SINGH, P.A. A general purpose laboratory diet mixture for rearing insects. Insect Science and its Application, v.4, p.357-362, 1983.

TZANAKAKIS, M.E.; PHILLIPS, J.H.H. Artificial diets for larvae of the Oriental fruit moth. Journal of Economic Entomology, v.62, p.879-882, 1969.

$\overline{\text { Recebido em } 19 \text { de maio de } 2010 \text { e aprovado em } 5 \text { de agosto de } 2010}$ 